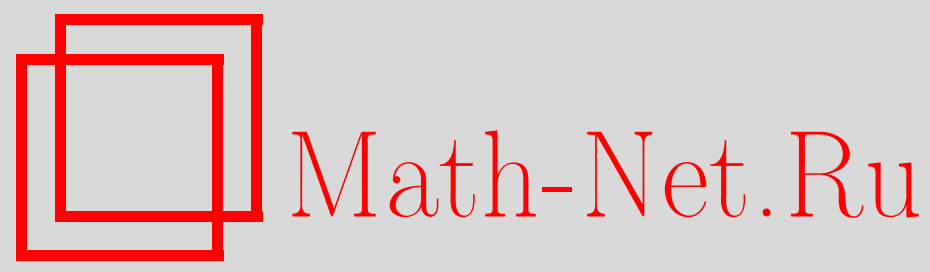

С. А. Антонян, О строго полусвободных действиях компактных групп Ли на гильбертовом кубе, Матем. заметки, 1999, том 65, выпуск 2, 163-174

DOI: https://doi.org/10.4213/mzm1038

Использование Общероссийского математического портала Math-Net.Ru подразумевает, что вы прочитали и согласны с пользовательским соглашением http://www . mathnet.ru/rus/agreement

Параметры загрузки:

IP: 3.85 .73 .92

26 апреля 2023 г., $17: 49: 02$

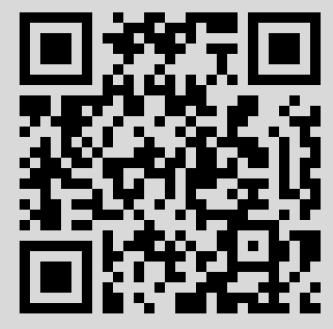




\section{О СТРОГО ПОЛУСВОБОДНЫХ ДЕЙСТВИЯХ КОМПАКТНЫХ ГРУПП ЛИ НА ГИЛЬБЕРТОВОМ КУБЕ}

\section{С. А. Антонян}

Доказано, что строго полусвободное действие $\alpha$ данной компактной группы Ли $G$ на гильбертовом кубе $Q$ эквивалентно стандартному строго полусвободному действию $\sigma$ тогда и только тогда, когда пространство орбит $Q_{0} / \alpha$ свободной части $Q_{0}=Q \backslash\{*\}$ является $Q$-многообразием и имеет собственный гомотопический тип пространства орбит $Q_{0} / \sigma$. Установлено существование эквивариантной ретракции $\left(Q_{0}, \sigma\right) \rightarrow\left(Q_{0}, \alpha\right)$. Доказано, что семейство всех эквивариантных отображений $X \rightarrow \operatorname{con} G$ для любого тихоновского $G$-пространства $X$ разделяет точки и замкнутые множества в $X$.

Библиография: 17 названий.

Введение. Одна из наиболее трудных и интересных областей бесконечномерной топологии касается вопросов действий компактных грушп на гильбертовом кубе $Q$ и смоделированных на нем многообразиях ( $Q$-многообразиях). Несмотря на усиленную активность, в этой области получено небольшое количество результатов. Дж. Вест заметил [1, с. 144], что любая компактная группа Ли $G$ может действовать строго полусвободно (т.е. с одной неподвижной точкой и свободно вне этой точки) на гильбертовом кубе $Q$ следующим образом: имеется естественное строго полусвободное действие $G$ на конусе $\operatorname{con} G$, порожденное левыми сдвигами группы $G$ на себя. Рассмотрим счетную степень $(\operatorname{con} G)^{\infty}$ и покоординатно определенное действие $G$ на ней, которое, очевидно, также является строго полусвободным. Заметим, что $(\operatorname{con} G)^{\infty}$ согласно результату Веста гомеоморфно гильбертову кубу $Q[2$, с. 62$]$, ибо $\operatorname{con} G$ есть AR-компакт. Это действие называется стандартным строго полусвободным действием (СПД).

До сих пор не решена проблема о единственности СПД на гильбертовом кубе [1], [3].

ПроБЛЕМА ЕДИНСТВенноСТИ. Пусть $G$ - компактная группа Ли, а $\alpha u \beta-$ два СПД группь $G$ на гильбертовом кубе $Q$. Верно ли, что эти действия эквивалентны, т.е. существует әквивариантный гомеоморфизм между $G$-пространствами $(Q, \alpha)$ u $(Q, \beta)$ ?

Первьй результат, относящийся к этой проблеме, получен Р. И. Вонгом [4].

КРИТЕРИЙ ВоНГА. Если $G=\mathbb{Z}_{p}$ - ииклическая группа и оба $G$-пространства $(Q, \alpha)$ и $(Q, \beta)$ обладают сколь угодным малыми стягиваемыми инвариантными окрестностями неподвижной точки, то СПД $\alpha$ и $\beta$ эквивалентны.

В [5] Л. Т. Ньюмэн доказал, что критерий Вонга не распространяется на произвольные $Q$-многообразия. В работе [3] И. Берштейн и Дж. Вест в случае произвольной компактной группы Ли $G$ получили ряд критериев эквивалентности данных СПД $\alpha$ и $\beta$ на 
гильбертовом кубе, сводя задачу к тонким вопросам собственной гомотопической теории. Напомним, что собственная гомотопическая теория от обычной отличается лишш тем, что в ней все отображения (в частности, все гомотопии) являются собственньми, т.е. отображениями, при которых прообраз любого компакта есть компакт.

Наиболее общий критерий из [3] формулируется следующим образом.

ТЕОРемА БЕРШТЕЙНА-ВЕСТА. Пусть $G$ - компактная группа Ли, $а \alpha u \beta-$ два ее СПД на гильбертовом кубе $Q$ с общей неподвижной точкой $a \in Q$. Пусть $Q_{0}=Q \backslash\{a\}, Q_{0} / \alpha$ является $Q$-многообразием и каждая гомотопическая әквивалентность $Q_{0} / \alpha \rightarrow Q_{0} / \alpha$ гомотопна некоторой собственной гомотопической әквивалентности $Q_{0} / \alpha \rightarrow Q_{0} / \alpha$. Тогда $\beta$ әквивалентно $\alpha$ в том и только том случае, когда $Q_{0} / \beta$ является $Q$-многообразием и собственно гомотопически эквивалентно $Q_{0} / \alpha$.

Как этот, так и все остальные критерии в [3] содержат следующую гипотезу.

ГИПОТЕЗА БЕРШТЕЙНА-ВЕСТА. Пусть $\alpha$ - СПД данной компактной группы Ли $G$ на гильбертовом кубе $Q$, а $Q_{0}$ - дополнение $\kappa$ неподвижной точке. Тогда пространство орбит $Q_{0} / \alpha$ есть $Q$-многообразие.

Очевидно, эта гипотеза верна, когда $G$ - конечная группа. Из теоремы 6 (см. ниже) следует, что гипотеза Берштейна-Веста верна для стандартного СПД любой компактной групшы Ли $G$. Отсюда и из теоремы Берштейна-Веста с учетом того, что для стандартного СПД $\sigma$ любая гомотопическая эквивалентность $Q_{0} / \sigma \rightarrow Q_{0} / \sigma$ гомотопна некоторой собственной гомотопической эквивалентности $Q_{0} / \sigma \rightarrow Q_{0} / \sigma[3]$, вытекает

ТЕорема 1. СПД $\alpha$ компактной группь Ли $G$ на гильбертовом кубе $Q$ әквивалентно стандартному СПД $\sigma$ тогда и только тогда, когда $Q_{0} / \sigma$ является $Q$-многообразием и имеет собственный гомотопический тип $Q_{0} / \sigma$.

Следствие 5, уточняя результат Берштейна и Веста [3], утверждает, что с помощью конструкции взятия произведения конечномерных AR-компактов нельзя получить нестандартное СПД на гильбертовом кубе.

Другой наш результат (теорема 5) утверждает, что для любого СПД $\alpha$ компактной группы Ли $G$ на кубе $Q \quad G$-пространство $\left(Q_{0}, \alpha\right)$ является строгим деформационньп $G$-ретрактом для $\left(Q_{0}, \sigma\right)$, где $\sigma$ - стандартное СПД на $Q$. Этот результат также вселяет некоторую надежду на положительное решение проблемы единственности СПД, ибо существование эквивариантной собственной ретракции $r:\left(Q_{0}, \sigma\right) \rightarrow\left(Q_{0}, \alpha\right)$ уже означает эквивалентность $\alpha$ и $\sigma$ (предложение 1).

На пути к доказательству этих результатов важную роль играет теорема 2, которая, как нам кажется, представляет и самостоятельньй интерес. Как известно, исключительно важная роль, которую играет отрезок $[0,1]$ в топологии, обусловлена прежде всего тем его свойством, что на "хороших" топологических пространствах сушествует “достаточно много" непрерьвных функций со значениями в [0,1]. До сих пор в теории $G$-пространств при решении конкретных задач [6]-[11] подобную роль в разных ситуациях играли разные компактные выпуклые инвариантные подмножества подходящих топологических линейных $G$-пространств. Единого же объекта, заменяющего отрезок $[0,1]$, в категории $G$-пространств не было найдено. Теорема 2 и ее следствие 1 как раз решают эту задачу, утверждая, что в категории $G$-пространств роль отрезка $[0,1]$ играет $\operatorname{con} G$ (случай компактной групшы Ли $G$ и свободных или строго полусвободных компактных 
$G$-пространств) и семейство конусов $\operatorname{con}(G / H)$ над всевозможными $G$-орбитами $G / H$, являющимися гладкими многообразиями (случай произвольной групы $G$ и всех тихоновских $G$-пространств). Отсюда вытекает существование универсальных тихоновских $G$-кубов (теорема 3 и следствие 2 ).

1. Предварительные определения и результаты. Всюду в работе, если не оговорено противное, предполагаем, что $G$ - произвольная компактная хаусдорфова группа. Все пространства предполагаются тихоновскими. Необходимые понятия и факты теории $G$-пространств можно найти в [6] и [7]. Используемые здесь понятия и результаты эквивариантной теории ретрактов содержатся в статьях [8]-[12].

В дальнейшем запись $X \in G \mathrm{AR}$ (соответственно $X \in G \mathrm{ANR}$ ) означает, что $X$ является эквивариантным абсолютным (соответственно окрестностньм) ретрактом для класса $M^{G}$ всех метризуемых $G$-пространств. Аналогично, запись $X \in G$ АЕ (соответственно $X \in G \mathrm{ANE}$ ) означает, что $X$ является эквивариантным абсолютным (соответственно окрестностньм) экстензором для класса $M^{G}$. Известно, что $G \mathrm{ANE} \cap M^{G}=$ $G \mathrm{ANR}$ и $G \mathrm{AE} \cap M^{G}=G \mathrm{AR}[9]$. Обозначим через $G \mathrm{AE}(N)($ соответственно $G \mathrm{ANE}(N))$ класс всех эквивариантных абсолютных (соответственно окрестностных) экстензоров для класса $N^{G}$ всех нормальных $G$-пространств. В дальнейшем понятие эквивариантного отображсени, или G-отображения в себя, будет включать также и его непрерьвность. Эквивариантный гомеоморфизм мы назьваем әквиморфизмом. Все встречающиеся в работе произведения $G$-пространств рассматриваются как $G$-пространства, наделенные покоординатно определенньм действием групш $G$.

Напомним, что если $X$ - произвольное $G$-пространство, а $x \in X$, то стабилизатором точки $x$ называется подгрупп $G_{x}=\{g \in G: g x=x\}$ группы $G$. Назовем $G$-пространство $X$ (соответственно действие на нем) свободным, если стабилизатор любой точки $x \in X$ тривиален, т.е. $G_{x}=\{e\}$ - единичная подгруппа. Назовем $G$-пространство $X$ (соответственно действие на нем) строго полусвободным , если в нем существует ровно одна точка $a \in X$, для которой $G_{a}=G$, и $G_{x}=\{e\}$ для любой отличной от $a$ точки $x \in X$. Если $H$ - замкнутая подгруппа $G$, то на факторпространстве $G / H$ левых смежных классов по $H$ група $G$ непрерьвно действует левыми сдвигами. Далее под $G / H$ всюду подразумевается именно это $G$-пространство.

ОПРЕДЕЛЕНИЕ 1. Замкнутую подгруппу $H<G$ назовем большой подгруппой, если существует замкнутая нормальная подгруппа $N$ групшы $G$ такая, что $N \subset H$ и $G / N$ есть группа Ли.

Нам потребуются две леммы, доказанные в [12].

Лемма 1. Если $H<G$ - большая подгруппа, то $G$-пространство $G / H$ является гладким многообразием и $G \mathrm{ANE}(N)$-пространством.

Лемма 2. Пусть $L<G$ - замкнутая подгруппа, а $U$-окрестность $L$ в $G$. Тогда существует большая подгруппа $K<G$ такая, ито $L \subset K \subset U$.

Лемма 3. Пусть $X$ - компактное GAR-пространство (соответственно GANR-пространство). тогда $X \in G \mathrm{AE}(N)$ (соответственно $X \in G \mathrm{ANE}(N))$.

ДокАЗАТЕЛЬСтво. Согласно [9] можно считать, что $X$ является инвариантньм подмножеством некоторого гильбертова $G$-куба $Q$. Так как $Q \in G \mathrm{AE}(N)$ (см. [9]), то $X$, будучи эквивариантным (соответственно окрестностным) ретрактом $Q$, само является $G \mathrm{AE}(N)$-пространством (соответственно $G \mathrm{ANE}(N)$-пространством). 
2. Эквивариантные вложения и продолжения. Напомним, что конусом над пространством $X$ назьвается факторпространство соn $X$ произведения $X \times[0,1]$ по его замкнутому подпространству $X \times\{0\}$. Класс точек $(x, t) \in X \times[0,1], t \neq 0$, обозначим через $t x$, а класс точек $(x, 0) \in X \times[0,1]$, называемьй вершиной конуса, - символом *.

Если $X$ является $G$-пространством, то легко проверить, что con $X$ превращается в $G$-пространство относительно следующего действия групы $G$ :

$$
g(t x)=t(g x), \quad g *=*,
$$

где $g \in G, t x \in \operatorname{con} X, t \neq 0$, a $*$ - вершина конуса.

Если $X$ - метризуемьй компакт, то con $X$ также является метризуемым компактом (см. $[13$, с. 177]).

ЛЕмма 4. Если $H<G-$ большая подгрупnа, mо $\operatorname{con}(G / H) \in G \operatorname{AE}(N)$.

ДокАЗАтЕльство. По лемме $1 G / H \in G \mathrm{ANR}$ и, тем более, $G / H$ локально $G$-стягиваемо [11]. Конус $\operatorname{con}(G / H)$ наследует от $G / H \times[0,1]$ его локальную $G$-стягиваемость и эквивариантно стягивается в свою вершину. Поскольку $\operatorname{con}(G / H)$ конечномерен, согласно [8] отсюда следует, что $\operatorname{con}(G / H) \in G$ AR. Осталось применить лемму 3.

Теорема 2. Пусть $X$ - произвольное $G$-пространство, $F$ - замкнутое подмножество $X$, а точка $a \in X$ не принадлежит $F$. Тогда существуют большая подгруппа $H<G$, содержащая в себе стабилизатор $G_{a}$, и әквивариантное отображение $f: X \rightarrow \operatorname{con}(G / H)$ такие, что $f(a) \notin \overline{f(F)}$. Более того, если $G_{a}-$ большая подгруппа, то можно положить $H=G_{a}$.

ДокАЗАтЕЛЬСтво. Пусть $U=\{g \in G: g a \in X \backslash F\}$. Ясно, что $U$ - открытая окрестность стабилизатора $G_{a}$ в групе $G$. Согласно лемме 2 сушествует большая подгруппа $H<G$ такая, что $G_{a} \subset H \subset U$. Если $G_{a}$ - большая подгруппа, то положим $H=G_{a}$. Определим отображение $\varphi: G(a) \rightarrow \operatorname{con}(G / H)$ формулой $\varphi(g a)=g H, g \in G$. Это определение корректно, ибо $G_{a} \subset H$. Ясно также, что $\varphi$ эквивариантно. Рассмотрим эквивариантное вложение $X$ в его некоторое компактное $G$-расширение $B$ (существование которого доказано в [14]). Так как $G(a)$ замкнуто в $B$ и $\operatorname{con}(G / H) \in G \mathrm{AE}(N)$ (лемма 4), отображение $\varphi$ допускает эквивариантное продолжение $\varphi_{1}: B \rightarrow \operatorname{con}(G / H)$. Рассмотрим сужение $\psi=\varphi_{1} \mid X$. Из включения $H \subset U$ следует, что $\varphi(a) \notin \varphi(F \cap G(a))$. Поскольку $\varphi(F \cap G(a))$ - компакт, можно подобрать окрестность $U_{1}$ точки $\psi(a)=\varphi(a)$ и окрестность $U_{2}$ множества $\psi(F \cap G(a))=\varphi(F \cap G(a))$ в $\operatorname{con}(G / H)$ такие, что $U_{1} \cap I U_{2}=\varnothing$, где $I U_{2}=\left\{t x \in \operatorname{con}(G / H): x \in U_{2}, t \in I\right\}, I=[0,1]$. Далее, рассмотрим окрестность $\psi^{-1}\left(U_{2}\right) \cup(X \backslash F)$ инвариантного множества $G(a)$ в $X$. В ней содержится инвариантная окрестность $W$ множества $G(a)[6$, с. 5$]$. Пусть $\alpha: X \rightarrow I$-инвариантная функция такая, что $G(a) \subset \alpha^{-1}(1), X \backslash W \subset \alpha^{-1}(0)$. Положим $f(x)=\alpha(x) \psi(x)$ для любого $x \in X$. Покажем, что $f$ - искомое отображение. Очевидно, что $f$ эквивариантно. Далее имеем $f(F \cap(X \backslash W))=*, f(a)=\alpha(a) \psi(a)=\varphi(a)=H$. Поэтому $f(a) \notin \overline{f(F \cap(X \backslash W))}$. С другой стороны, $f(F \cap W) \subset f\left(F \cap \psi^{-1}\left(U_{2}\right)\right) \subset f\left(\psi^{-1}\left(U_{2}\right)\right) \subset I U_{2}$. Так как $U_{1}$ непересекается с $I U_{2}$, отсюда заключаем, что $f(a) \notin \overline{f(F \cap W)}$. Таким образом, $f(a) \notin \overline{f(F)}$ и теорема доказана. 
СЛЕДСТВИЕ 1. Если $G$ - группа Ли, $а X$ - произвольное свободное (соответственно строго полусвободное компактное) G-пространство, то множество всех әквивариантных отображсенй $X \rightarrow \operatorname{con} G$ разделяет точки и замкнутые множества (соответственно пары точек) в $X$ в смысле теоремы 2.

Следующий результат является эквивариантным аналогом известной теоремы Тихонова о вложении в куб $I^{\tau}=[0,1]^{\tau}$.

ТЕОрема 3. Пусть $\tau$ - бесконечный кардинал, $a G-$ группа веса $w G \leqslant \tau$. Тогда существует семейство $\left\{H_{j}: j \in J\right\}$ больиих подгрупп $H_{j}<G$ такое, что

1) мощность $|J| \leqslant \tau$;

2) для каждого G-пространства $X$ веса $w X \leqslant \tau$ существует эквиморфное влохсение $X \rightarrow \prod_{j \in J} \operatorname{con}\left(G / H_{j}\right)$;

3) произведение $\prod_{j \in J} \operatorname{con}\left(G / H_{j}\right)$ гомеоморфно тихоновскому кубу $I^{|J|}$ иявляется $G \mathrm{AE}(N)$-пространством.

ДоКАЗАТЕЛЬСтво. Рассмотрим $G$-пространство $C\left(G, I^{\tau}\right)$ всех непрерывных отображений $f: G \rightarrow I^{\tau}$, наделенное компактно открытой топологией и действием $(g f)(t)=$ $f(t g)$, где $g, t \in G, f \in C\left(G, I^{\tau}\right)$. Согласно теореме 2 множество $\Phi$ всех эквивариантных отображений $\varphi: C\left(G, I^{\tau}\right) \rightarrow \operatorname{con}(G / H)$ во всевозможные конусы $\operatorname{con}(G / H)$ с большой подгрупой $H$ разделяет точки и замкнутые множества в $C\left(G, I^{\tau}\right)$. Поскольку вес $w\left(C\left(G, I^{\tau}\right)\right)=\tau$, согласно хорошо известному приему можно выбрать подмножество $J \subset \Phi$ мощности $\tau$, также разделяющее точки и замкнутые множества. Мы утверждаем, что множество $\left\{H_{j}: j \in J\right\}$ всех больших подгрупп $H_{j}<G$, соответствующих отображениям $\varphi_{j}: C\left(G, I^{\tau}\right) \rightarrow \operatorname{con}\left(G / H_{j}\right)$ из множества $J$, искомое. Действительно, свойство 1) выполнено. Свойство 3$)$ следует из того, что $\operatorname{con}\left(G / H_{j}\right) \in \mathrm{AR}$, и из результата Веста [2, с. 62] о том, что счетное произведение неодноточечных AR-компактов гомеоморфно гильбертову кубу. Для проверки свойства 2) рассмотрим диагональное произведение $\Delta J$ всех отображений семейства $J$. Поскольку $J$ разделяет точки и замкнутые множества, $\Delta J: C\left(G, I^{\tau}\right) \rightarrow \prod_{j \in J} \operatorname{con}\left(G / H_{j}\right)$ является топологическим вложением. Его эквивариантность следует из эквивариантности всех отображений $\varphi_{j} \in J$. Для завершения доказательства осталось показать, что каждое $G$-пространство $X$ веса $w X \leqslant \tau$ эквивариантно вкладьвается в $C\left(G, I^{\tau}\right)$. Для этого достаточно взять вложение $i: X \rightarrow I^{\tau}$ и определить отображение $s: X \rightarrow C\left(G, I^{\tau}\right)$ по правилу $s(x)(g)=i(g x)$, где $x \in X, g \in G$. Несложно проверить, что $s$ - эквивариантное вложение. Теорема доказана.

Совершенно аналогично с использованием следствия 1 доказьвается

СЛЕДСТВИЕ 2. Пусть $G$ - әруппа Ли, a $\tau$ - произвольный бесконечный кардинал. Тогда любое свободное (соответственно строго полусвободное компактное) $G$-пространство $X$ обладает әквивариантным вложением в строго полусвободное $G$-пространство $(\operatorname{con} G)^{\tau}$.

ЗАмЕчАниЕ 1 . В следствии 2 , на самом деле, свободное $G$-пространство $X$ эквиморфно вкладывается в свободное $G$-пространство $(\operatorname{con} G)^{\tau} \backslash\{*\}$, где *- единственная неподвижная (относительно действия) точка $G$-пространства $(\operatorname{con} G)^{\tau}$. Это следует из того, что при эквиморфизме сохраняется стабилизатор любой точки. Если $\tau=\infty-$ счетный кардинал, то $(\operatorname{con} G)^{\infty}$ гомеоморфно гильбертову кубу и $(\operatorname{con} G)^{\infty} \in G \mathrm{AR}$. 
Следовательно, свободное $G$-пространство $Q_{0}=(\operatorname{con} G)^{\infty} \backslash\{*\}$ является свободным $G$ ANR-пространством, универсальным в классе всех сепарабельных метризуемых свободных $G$-пространств. Очевидно, что $Q_{0}$, не имея $G$-неподвижных точек, не является GAR-пространством.

Однако, имеет место

Tеорема 4. Пусть G - группа Ли, свободно действующая на AR-пространстве $X$. Тогда G-пространство $X$ является GAE-пространством для класса всех локально компактных свободных $G$-пространств со счетной базой.

Для доказательства нам понадобится

ОПРЕДЕЛЕНИЕ 2. Скажем, что $G$-пространство $X$ имеет евклидов тип, если сушествует изовариантное отображение $f: X \rightarrow E$ в некоторое евклидово $G$-пространство $E$, т.е. $f$ эквивариантно и $G_{x}=G_{f(x)}$ для всех $x \in X$.

ДОКАЗАТЕЛЬСТво ТЕОРЕМЫ 4. Пусть $Y$ - любое локально компактное свободное $G$-пространство со счетной базой, $A$ - произвольное замкнутое инвариантное подмножество $Y$, а $f: A \rightarrow X$ - эквивариантное отображение. Хорошо известно, что $Y$ можно представить в виде $Y=\bigcup_{n=1}^{\infty} U_{n}$, где $U_{n} \subset Y$ - открытое подмножество с компактным замьканием $F_{n}$ и $F_{n} \subset U_{n+1}$ для всех $n=1,2, \ldots$ Можно также считать, что каждое $U_{n}$ инвариантно относительно действия группы $G$ (в противном случае можно перейти от $U_{n}$ к его орбите $\left.G\left(U_{n}\right)=\bigcup_{g \in G} g U_{n}\right)$.

Положим $A_{n}=A \cup F_{n}$ для каждого $n=1,2, \ldots$ Ясно, что $A_{n}$ - замкнутое инвариантное подмножество $Y$, и дополнение $A_{n} \backslash A$, будучи инвариантньм подмножеством компактного свободного $G$-пространства $F_{n}$, является $G$-пространством евклидова типа, как это показал Я. Яворовский [15]. Поскольку $X \in \mathrm{AR}$, согласно результату Яворовского [15] существует эквивариантное продолжение $f_{1}: A_{1} \rightarrow X$ отображения $f: A \rightarrow X$. Точно так же отображение $f_{1}$ продолжается до эквивариантного отображения $f_{2}: A_{2} \rightarrow X$, и т.д. Таким образом, получаем последовательность $\left\{f_{n}: A_{n} \rightarrow X\right\}$ эквивариантных отображений такую, что $f_{n+1}$ является продолжением $f_{n}$. Теперь отображение $\varphi: Y \rightarrow X$ определим по правилу $\varphi(x)=f_{n}(x)$ для всех $x \in F_{n}$. Ясно, что $\varphi$ корректно определено и его непрерьвность следует из представления $Y=\bigcup_{n=1}^{\infty} U_{n}$. Итак, $\varphi$ - искомое эквивариантное продолжение отображения $f$ и теорема доказана.

СлЕДСТВИЕ 3. Пусть на $G$-пространстве $X$ әруппа $G$ действует свободно $u$ $X \in \mathrm{AR}$. Тогда для любого локально компактного свободного $G$-пространства $Y$ со счетной базой любые два эквивариантных отображсени из $Y$ в $X$ әквивариантно гомотопны.

3. Строго полусвободные действия на гильбертовом кубе. Пусть $G$ - произвольная компактная группа Ли. Всюду далее обозначаем через $Q$ гильбертов куб (рассматриваемый как $G$-пространство), наделенный стандартньм СПД групшы $G$, а через $Q_{0}$ свободную часть $Q$, т.е. дополнение к единственной $G$-неподвижной точке в $Q$. Обозначим через $\widetilde{Q}$ гильбертов куб, наделенньй произвольным СПД групшы $G$, а через $\widetilde{Q}_{0}$ свободную часть $\widetilde{Q}$. Очевидно, вопрос об эквиморфности $G$-пространств $Q$ и $\widetilde{Q}$ (т.е. об эквивалентности соответствующих СПД) равносилен вопросу об эквиморфности их свободных частей $Q_{0}$ и $\widetilde{Q}_{0}$. Это проблема единственности СПД, которая не решена даже в случае группы $G=\mathbb{Z}_{2}$. 
В этом пункте мы приводим некоторые новые результаты, относящиеся к этой проблеме.

ТеОРема 5. G-пространство $\widetilde{Q}_{0}$ является строгим деформационным G-ретрактом для G-пространства $Q_{0}$. В частности, $\widetilde{Q}_{0}$ и $Q_{0}$ имеют одинаковый G-гомотопический тuп.

ДокАЗАТЕльство. Согласно следствию $2 G$-пространство $\widetilde{Q}$ можно считать инвариантньп подпространством $G$-пространства $Q$. Поскольку при эквиморфном вложении сохраняется стабилизатор любой точки, то $\widetilde{Q}_{0}=Q_{0} \cap \widetilde{Q}$. Таким образом, $\widetilde{Q}_{0}-$ замкнутое инвариантное подмножество в $Q_{0}$. Согласно теореме 4 тождественное отображение $\widetilde{Q}_{0} \rightarrow \widetilde{Q}_{0}$ допускает эквивариантное продолжение $r: Q_{0} \rightarrow \widetilde{Q}_{0}$.

Рассмотрим произведение $Q_{0} \times I$ и его замкнутое инвариантное подмножество

$$
A=Q_{0} \times\{0\} \cup \widetilde{Q}_{0} \times I \cup Q_{0} \times\{1\} .
$$

Определим отображение $f: A \rightarrow Q_{0}$ по правилу $f(x, 0)=r(x), f(x, 1)=x, f(a, t)=a$, где $x \in Q_{0}, a \in \widetilde{Q}_{0}, t \in I$. Ясно, что $f$ - эквивариантное отображение. Повторно применяя теорему 4, мы получим эквивариантное продолжение $F: Q_{0} \times I \rightarrow Q_{0}$ отображения $f$, которое и есть искомая строгая деформационная $G$-ретракция. В частности, ретракция $r: Q_{0} \rightarrow \widetilde{Q}_{0}$ является эквивариантной гомотопической эквивариантностью.

Переходя к пространствам орбит, непосредственно из теоремы 5 получим

СлЕдСТВИЕ 4. Пространство $\widetilde{Q}_{0} / G$ является строгим деформационным ретрактом для $Q_{0} / G$. В частности, они имеют одинаковый гомотопический тип.

ПРЕДЛОЖЕНИЕ 1. Для конечной әруппь $G$ следующие утверждения попарно эквивалентны:

1) $\underset{Q}{\sim} \widetilde{Q}$ әквиморфны;

2) $\widetilde{Q} \in G \mathrm{AR}$;

3) $\widetilde{Q}_{0}$ является собственным әквивариантным ретрактом $Q_{0}$, т.е. существует такая $G$-ретракиия $Q_{0} \rightarrow \widetilde{Q}_{0}$, при которой прообраз любого компакта есть компакт.

ДоКАЗАТЕЛЬСтво. Импликация 1 ) $\Longrightarrow 2$ ) следует из того, что con $G \in G$ AR (лем-

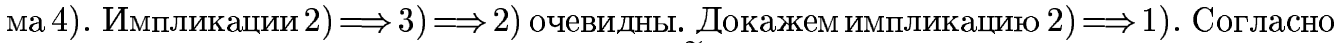
результату автора [10] пространство орбит $\widetilde{Q} / G$ есть AR-пространство. Осталось воспользоваться результатом Веста и Вонга [16].

Tеорема 6. Пусть $\left\{X_{i}: i=1,2, \ldots\right\}$ - семейство метризуемых GAR-компактов, $a_{i} \in X_{i}, i=1,2, \ldots$, - некоторая фиксированная $G$-неподвижная точка. Предполохсим, что выполняется условие:

i) для любых $x_{i} \in X_{i}, i=1,2, \ldots$, существует бесконечно много индексов $j=j(i)$, для каждого из которых найдется отличная от $a_{j}$ точка $b_{j} \in X_{j}$ такая, ито $G_{x_{i}} \subset G_{b_{j}}$.

Пусть далее $a=\left\{a_{i}\right\}_{i=1}^{\infty}, X=\left(\prod_{i=1}^{\infty} X_{i}\right) \backslash\{a\}$. Тогда пространство орбит $L=X / G$ является $Q$-многообразием.

Доказательство распадается на несколько лемм. 
Для пространств $T$ и $Z$ обозначим через $C(T, Z)$ пространство всех непрерьвных отображений $T \rightarrow Z$, наделенное компактно открытой топологией. Для $G$-пространств $T$ и $Z$ обозначим через $E(T, Z)$ подпространство $C(T, Z)$, состоящее из всех эквивариантных отображений $T \rightarrow Z$.

ОПРЕДЕЛЕНИЕ 3. Замкнутое инвариантное подмножество $A$ данного $G$-пространства $X$ назовем $G Z$-мно жеством, если для любого $G$-компакта $K$ в пространстве $E(K, X)$ всюду плотно его подмножество $\{f \in E(K, X): f(K) \cap A=\varnothing\}$.

Лемма 5. Если $A$ есть $G Z$-множество в метризуемом $G$-пространстве $X$, то $A / G$ есть $Z$-множество в $X / G$.

ДокАЗАТЕЛЬСТво. Пусть $M$ - произвольный компакт. Необходимо показать [17], что в пространстве $C(M, X / G)$ всюду плотно его подмножество $\{\varphi \in C(M, X / G)$ : $\varphi(M) \cap(A / G)=\varnothing\}$. Рассмотрим некоторую $G$-инвариантную метрику $\rho$ на $X$. Тогда, как известно, топология пространства орбит $X / G$ задается метрикой

$$
\rho^{*}(G(x), G(y))=\inf _{g \in G} \rho(x, g y), \quad G(x), G(y) \in X / G
$$

Очевидно, что

$$
\rho^{*}(G(x), G(y)) \leqslant \rho(x, y) .
$$

Возьмем произвольньй элемент $\varphi \in C(M, X / G)$ и любое число $\varepsilon>0$. Пусть $p: X \rightarrow$ $X / G$ - орбитное отображение. Тогда известно [7, с. 56], что сушествует коммутативная диаграмма (послойное произведение)

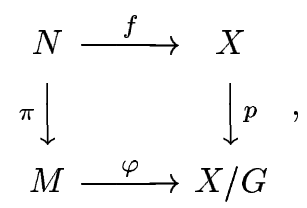

где $N$ - компактное $G$-пространство, $N / G=M, \pi: N \rightarrow M$ - орбитное отображение, а $f$ - эквивариантное отображение, индуцирующее отображение $\varphi$. Поскольку $N$ является $G$-компактом и $A \subset X$ есть $G Z$-множество, существует эквивариантное отображение $l: N \rightarrow X$ такое, что $l(N) \cap A=\varnothing$ и $\rho(l(n), f(n))<\varepsilon$ для всех $n \in N$. Рассмотрим индуцированное отображением $l$ отображение $\psi: N / G \rightarrow X / G$ пространств орбит. Пусть $m \in M$ - любая точка, а $n \in N$ - точка такая, что $\pi(n)=m$. Тогда из коммутативности диаграммы (2) следует, что $\psi(m)=p(l(n)), \varphi(m)=p(f(n))$. Отсюда согласно $(1)$ и выбору $l$ следует, что $\rho^{*}(\psi(m), \varphi(m)) \leqslant \rho(l(n), f(n))<\varepsilon$. Для завершения доказательства осталось заметить, что

$$
\psi(M) \cap(A / G)=p(l(N) \cap A)=p(\varnothing)=\varnothing .
$$

Лемма 6. Пусть G-неподвижная точка $a \in \prod_{i=1}^{\infty} X_{i}$ имеет координаты $a_{i} \in X_{i}$, $i=1,2, \ldots, u k$ - фиксированное натуральное число. Тогда множество $A=\{x \in X$ : $\left.x_{i}=a_{i}, i \geqslant k\right\}$ является $G Z$-мнохеством в $G$-пространстве $X=\left(\prod_{i=1}^{\infty} X_{i}\right) \backslash\{a\}$. 
ДокАЗАтЕльство. Ясно, что $A$ - замкнутое инвариантное подмножество $X$. Пусть $f: N \rightarrow X$-произвольное эквивариантное отображение данного $G$-компакта $N$, а $\varepsilon>0$ - любое число. Пусть номер $d$ выбран так, что $d>k$ и

$$
\sum_{i=d}^{\infty} \frac{1}{2^{i}}<\varepsilon .
$$

Пусть $\rho_{i}$ - метрика на $X_{i}$ с диаметром, не превосходящим 1. На произведении $\prod_{i=1}^{\infty} X_{i}$ рассмотрим метрику

$$
\rho(x, y)=\sum_{i=1}^{\infty} \frac{\rho_{i}\left(x_{i}, y_{i}\right)}{2^{i}}
$$

где $x=\left\{x_{i}\right\}, y=\left\{y_{i}\right\} \in \prod_{i=1}^{\infty} X_{i}$. Пусть $f(x)=\left\{f_{i}(x)\right\}_{i=1}^{\infty}, x \in N$. Установим, что можно построить семейство эквивариантных отображений $\left\{\varphi_{i}: N \rightarrow X_{i}, i \geqslant d+1\right\}$ такое, что для каждой точки $x \in N$ найдется хотя бы один индекс $j \geqslant d+1$ такой, что $\varphi_{j}(x)=a_{j}$. Для этого вокруг каждой орбиты $G(x) \subset N$ рассмотрим ее некоторую инвариантную трубчатую окрестность $U_{x}$, существование которой обеспечивается теоремой Мостова [7, с. 93]. Другими словами, “трубчатость” окрестности $U_{x}$ означает существование эквивариантной ретракции $r_{x}: U_{x} \rightarrow G(x)$ на орбиту $G(x)[7$, c. 95]. Очевидно, инвариантную окрестность $U_{x}$ можно считать замкнутой. В силу компактности $N$ его покрытие $\left\{U_{x}: x \in N\right\}$ обладает конечным подпокрытием $\left\{U_{x_{1}}, \ldots, U_{x_{n}}\right\}$. Теперь для каждого $i=1, \ldots, n$ определим $G$-отображение $q_{i}: U_{x_{i}} \rightarrow X_{j(i)}$ следующим образом. Сначала рассмотрим точку $f\left(x_{1}\right)=\left\{y_{m}\right\} \in X$. Поскольку $f\left(x_{1}\right) \neq a$, то $y_{m} \neq a_{m}$ для некоторого индекса $m \geqslant 1$. Согласно условию i) из теоремы 6 существуют индекс $j(1)>d$ и точка $b_{j(1)} \in X_{j(1)}, b_{j(1)} \neq a_{j(1)}$, такие, что $G_{y_{m}} \subset G_{b_{j(1)}}$. Поскольку $G_{x_{1}} \subset G_{f\left(x_{1}\right)} \subset G_{y_{m}}$, то $G_{x_{1}} \subset G_{b_{j(1)}}$. Поэтому существует эквивариантное отображение $p_{1}$ орбиты $G\left(x_{1}\right) \subset N$ в орбиту $G\left(b_{j(1)}\right) \subset X_{j(1)}$. Полагая $q_{1}=r_{x_{1}}$ о $p_{1}$, где $r_{x_{1}}: U_{x_{1}} \rightarrow G\left(x_{1}\right)$ - эквивариантная ретракция, соответствующая трубчатой окрестности $U_{x_{1}}$, мы получаем эквивариантное отображение $q_{1}: U_{x_{1}} \rightarrow X_{j(1)}$, для которого $q_{1}(x) \neq a_{j(1)}$ для всех $x \in U_{x_{1}}$. Поскольку $U_{x_{1}}$ - замкнутое $G$-подмножество в $N$, а $X_{j(1)} \in G \mathrm{AR}$, то $q_{1}$ допускает эквивариантное продолжение $\varphi_{j(1)}: N \rightarrow X_{j(1)}$. Аналогично можно построить эквивариантное отображение $\varphi_{j(2)}: N \rightarrow X_{j(2)}$ такое, что $\varphi_{j(2)}(x) \neq a_{j(2)}$ для всех $x \in U_{x_{2}}$. Исходя из условия i) теоремы 6 , можно считать, что индексы $j(1)$ и $j(2)$ разные. Действуя аналогичным образом, на $n$-м шаге мы построим все $G$-отображения $\varphi_{j(i)}: N \rightarrow X_{j(i)}, i=1, \ldots, n$, такие, что $\varphi_{j(i)}(x) \neq a_{j(i)}$ для всех $x \in U_{x_{j(i)}}$ и все индексы $j(1), j(2), \ldots, j(n)$ разные. Для индексов $s>d$, отличных от индексов $j(1), j(2), \ldots, j(n), G$-отображение $\varphi_{s}: N \rightarrow X_{s}$ определим по правилу $\varphi_{s}(x)=a_{s}, x \in N$. Семейство отображений $\left\{\varphi_{i}: i \geqslant d+1\right\}$ искомое. Действительно, пусть $x \in N$ - любая точка. Тогда $x \in U_{x_{i}}$ для некоторого $i=1, \ldots, n$. А по построению $\varphi_{j(i)}(x)=q_{i}(x) \neq a_{j(i)}$. Таким образом, семейство эквивариантных отображений построено.

Теперь определим новое эквивариантное отображение $f^{\prime}: N \rightarrow X$ по правилу

$$
f^{\prime}(x)=\left(f_{1}(x), \ldots, f_{d}(x), \varphi_{d+1}(x), \varphi_{d+2}(x), \ldots\right), \quad x \in N .
$$

Ясно, что $f^{\prime}-$ корректно определенное эквивариантное отображение. Поскольку $d>k$ и среди $\varphi_{d+1}(x), \varphi_{d+2}(x), \ldots$ есть $\varphi_{d+i}(x)$ такое, что $\varphi_{d+i}(x) \neq a_{d+i}$, то $f^{\prime}(x) \notin A$ 
для любого $x \in N$. Таким образом, $f^{\prime}(N) \cap A=\varnothing$. Для завершения доказательства осталось заметить, что согласно выбору номера $d$ имеет место неравенство

$$
\rho\left(f(x), f^{\prime}(x)\right)=\sum_{i=d+1}^{\infty} \frac{\rho_{i}\left(f_{i}(x), \varphi_{i}(x)\right)}{2^{i}}<\varepsilon
$$

для всех точек $x \in N$.

Справедливость следующей леммы очевидна.

Лемма 7. Если $A$ является $G Z$-множеством в $G$-пространстве $Y$, а $B \subset Y$ есть замкнутое инвариантное подмножество, лежсащее в $A$, то $B$ также является $G Z$-множеством.

Лемма 8. Для любого компакта $M$ множество всех $Z$-отображений $q: M \rightarrow$ $X / G$ всюду плотно в $C(M, X / G)$ (X есть $G$-пространство из теоремы 6$)$.

ДокАЗАТЕЛЬСтво. Пусть $\varphi: M \rightarrow X / G$ - любое непрерьвное отображение, а $p:$ $X \rightarrow X / G$ - орбитное отображение. Как и в доказательстве леммы 5 (для удобства мы используем обозначения, принятые в этой лемме), рассмотрим диаграмму (2). Пусть $f: N \rightarrow X$ и компакт $f(N)$ лежит в $X=\left(\prod_{i=1}^{\infty} X_{i}\right) \backslash\{a\}$. Легко видеть, что существует номер $n$, удовлетворяющий следующему условию:

ii) для всякой точки $x \in X$ найдется индекс $i=1, \ldots, n$ такой, что $f_{i}(x) \neq a_{i}$ (в противном случае найдется последовательность $\left\{x_{k}\right\} \subset N$ такая, что $f\left(x_{k}\right) \rightarrow a$, откуда следует, что $a \in f(N))$.

Пусть $\varepsilon>0$ - любое число, а $k$ - натуральное число такое, что $k>n$ и

$$
\sum_{i=k}^{\infty} \frac{1}{2^{i}}<\varepsilon
$$

Определим эквивариантное отображение $s: N \rightarrow X$ по правилу

$$
s(x)=\left(f_{1}(x), \ldots, f_{k}(x), a_{k+1}, \ldots\right), \quad x \in N,
$$

где $f_{i}: N \rightarrow X_{i}$ - координатные функции отображения $f$ из диаграммы (2). Согласно выбору $k$ и условию ii) $s(x) \neq a$ для всех $x \in N$, т.е. отображение $s$ корректно определено. Далее имеем

$$
\rho(s(x), f(x))=\sum_{i=k+1}^{\infty} \frac{\rho_{i}\left(a_{i}, f_{i}(x)\right)}{2^{i}}<\varepsilon .
$$

Итак, эквивариантные отображения $f$ и $s \quad \varepsilon$-близки и множество $s(N)$ лежит в подмножестве $A=\left\{x \in X: x_{i}=a_{i}, i \geqslant k+1\right\}$ произведения $\prod_{i=1}^{\infty} X_{i}$. Из лемм 6 и 7 следует, что $s(N)$ является $G Z$-множеством. Утверждаем, что отображение $q: N / G \rightarrow X / G$, индуцированное $G$-отображением $s$, является $Z$-отображением, т.е. образ $q(M)$ есть $Z$-множество в $X / G$. Действительно, $q(M)=s(N) / G$ и $s(N)$ является $G Z$-множеством. Осталось применить лемму 5. Далее, пусть $m \in M$ - любая точка, а $n \in N$ - точка такая, что $\pi(n)=m$. Тогда $q(m)=p(s(n)), \varphi(m)=p(f(n))$. Отсюда согласно (1) и (3) следует, что $\rho^{*}(q(m), \varphi(m)) \leqslant \rho(s(n), f(n))<\varepsilon$ для всех $m \in M$. Таким образом, $q$ является $Z$-отображением, $\varepsilon$-близким к отображению $\varphi$. Лемма доказана. 
ДоКАЗАТЕЛЬСТВО ТЕОРЕМЫ 6. Будучи открытым инвариантным подмножеством $G$ AR-пространства $\prod_{i=1}^{\infty} X_{i}$, само $X$ является $G$ ANR-пространством. Согласно результату из [10] отсюда следует, что $X / G$ есть ANR-пространство. Осталось воспользоваться леммой 8 и применить известный критерий Торунчика [17], предварительно заметив, что $X / G$ является локально компактньм сепарабельным метризуемым пространством.

ЗАмЕЧАнИЕ 2. Условие і) из теоремы 6 очевидным образом выполняется, когда все $G$-пространства $X_{i} \backslash\left\{a_{i}\right\}$ имеют одинаковое множество орбитных типов, в частности, когда все $X_{i} \backslash\left\{a_{i}\right\}$ свободны. Поэтому из теоремы 6 непосредственно вытекает решение гипотезы Берштейна-Веста для стандартного СПД на гильбертовом кубе.

СлеДСТВИЕ 5. Пусть $Q$ - әильбертов куб, наделенный стандартным СПД компактной группь Ли $G, u Q_{0}$ - свободная часть $Q$. Тогда пространство орбит $Q_{0} / G$ является $Q$-многообразием.

ДокАЗАТЕЛЬСТво. По определению $Q=(\operatorname{con} G)^{\infty}$ и согласно лемме 4 con $G \in G$ AR. Осталось применить теорему 6.

СЛЕДСТВИЕ 6. Пусть $\left\{X_{i}: i=1,2, \ldots\right\}$ - счетное семейство конечномерных метризуемых невырожсденых $\mathrm{AR}$-компактов, на каждом из которых данная группа Ли $G$ действует строго полусвободно. Тогда диагональное действие $G$ на произведении $\prod_{i=1}^{\infty} X_{i}$ әквивалентно стандартному СПД на гильбертовом кубе $Q$.

ДокАЗАТЕЛЬСтво. Сначала воспользуемся результатом Яворовского [15], согласно которому конечномерноеметризуемое $G$-пространство $Y$ является $G$ AR-пространством, коль скоро множество всех $H$-неподвижных точек $Y[H]=\{y \in Y: g y=y, g \in G\}$ является AR-пространством для любой замкнутой подгруппы $H<G$. Согласно этому критерию каждое конечномерное метризуемое строго полусвободное AR-пространство $X_{i}, i=1,2, \ldots$, является $G \mathrm{AR}$-пространством. Следовательно, вьполняются условия теоремы 6 , согласно которой пространство орбит свободной части произведения $\prod_{i=1}^{\infty} X_{i}$ есть $Q$-многообразие. Применение теоремы 8.3 из [3] завершает доказательство.

В заключение сформулируем два вопроса, тесно связанные с известной гипотезой Гильберта-Смита о том, что компактная группа, свободно действующая на многообразии, непременно является группой Ли.

Вопрос 1. Верно ли, что компактная группа, строго полусвободно действующая на гильбертовом кубе, является групой Ли?

Вопрос 2. Верно ли, что компактная группа, свободно действующая на локально компактном (компактном) ANR-пространстве, является группой Ли?

ЗАМЕЧАНИЕ 3 . Пример 3 из [1, с. 144] строго полусвободного действия любой компактной нелиевой группы на гильбертовом кубе ошибочен (в этом совсем нетрудно убедиться). 


\section{СПИСОК ЦИТИРОВАННОЙ ЛИТЕРАТУРЫ}

[1] Чепмэн Т. А. Лекции о $Q$-многообразиях. М.: Мир, 1981.

[2] Федорчук В. В., Чигогидзе А. Ч. Абсолютные ретракты и бесконечномерные многообразия. М.: Наука, 1992.

[3] Berstein I., West J. E. Based free compact Lie group actions on Hilbert cubes // Proc. Sympos. Pure Math. Algebraic and Geometric Topology. 1987. V. 32. Part 1. P. 373-391.

[4] Wong R. Y.-T. Periodic actions on the Hilbert cube // Fund. Math. 1974. V. 85. № 3. P. 203-210.

[5] Newman L.S. J. Applications of group actions of finite complexes to Hilbert cube manifolds // Proc. Amer. Math. Soc. 1977. V. 62. № 1. P. 183-187.

[6] Palais R. The classification of $G$-spaces. Mem. Amer. Math. Soc. V. 36. Providence (R.I.): Amer. Math. Soc., 1960.

[7] Бредон Г. Введение в теорию компактных групп преобразований. М.: Наука, 1980.

[8] Antonian S. A. An equivariant theory of retracts // London Math. Soc. Lecture Note Ser. 1985. V. 93. P. 251-269.

[9] Antonian S. A. Equivariant embeddings into G-AR's // Glas. Mat. Ser. III. 1987. V. 22. № 1. P. 503-533.

[10] Антонян С. А. Ретракционные свойства пространства орбит // Матем. сб. 1988. T. 137 (179). № 3. C. 300-318.

[11] Антонян С. А. Ретракты в категориях $G$-пространств // Изв. АН АрмССР. Сер. матем. 1980. T. 15. № 5. C. 365-378.

[12] Антонян С. А. Существование среза для произвольной компактной группы преобразований // Матем. заметки. 1994. Т. 56. № 5. С. 3-9.

[13] Hu S.-T. Theory of Retracts. Detroit: Weyne State Univ. Press, 1965.

[14] de Vries J. On the existence of the $G$-compactifications // Bull. Acad. Polon. Sci. Ser. Math. Astr. Phys. 1978. V. 26. P. 275-280.

[15] Jaworowski J. An equivariant extension theorem and $G$-retracts with finite structure // Manuscripta Math. 1981. V. 35. № 3. P. 323-329.

[16] West J.E., Wong R. Y.-T. Based free actions of finite groups on Hilbert cubes with absolute retract orbit spaces are conjugate // Geometric Topology. Proc. 1977 Georgia Topology Conf. London: Acad. Press, 1979. P. 655-672.

[17] Torunczyk H. On $C E$-images of Hilbert cube and characterization of $Q$-manifolds // Fund. Math. 1980. V. 106. № 1. P. 31-40. 